\title{
Contribuições das Epistemologias de Kuhn e Fleck para a Reforma do Ensino Médico
}

PALAVRAS-CHAVE:

-Educação Médica;

-Filosofia Médica;

-Pensamento;

-Modelos Educacionais;

- Atenção Primária à Saúde;

- Medicina de Familia e Comunidade.

\section{KEY-WORDS:}

-Education, Medical;

-Philosophy, Medical;

-Thinking,

-Models, Educational;

-Primary Health Care;

-Family Practice.

Recebido em: 06/11/2006

Reenviado em: 03/07/2007

Aprovado em: 20/07/2007

\author{
Contributions of Kuhn's and Fleck's \\ Epistemology to the Reform of Medical \\ Teaching
}

Charles Dalcante Tesser ${ }^{1}$
O objetivo deste artigo é apresentar algumas idéias epistemológicas de Thomas Kuhn e Ludwik Fleck e correlacioná-las entre si e com os desafios da reforma da educação médica, supondo para esta uma finalidade de formação de médicos gerais habilitados, competentes e dispostos para o trabalho no SUS e no PSF (atenção básica). Os conceitos de paradigma e de ciência normal e a visão kuhniana do aprendizado científico são coerentes com os conceitos fleckianos de estilo de pensamento e iniciação nos círculos esotéricos de um coletivo de pensamento. Ambos os autores se reforçam na pertinência da aplicação dessas categorias à medicina e ao seu ensino. Indicam para este ensino direções coerentes com a inserção precoce dos alunos na atenção básica e com o aprendizado de práticas e habilidades ambientadas nesses serviços, ensinadas por profissionais que ali pratiquem medicina geral. Além disso, suas teorias facilitam o reconhecimento e o manejo das "reduções paradigmáticas" ou "estilísticas", melhorando a habilidade clínica e a performance ética dos profissionais e alunos.

\section{ABSTRACT}

The purpose of this article is to present some epistemological ideas of Thomas Kuhn and Ludwik Fleck correlating them to each other and to medical knowledge and practice, especially with regard to the challenges posed by the reform of medical education. We assume here that the purpose of medical education is preparing competent general practitioners, willing to work in primary care in the Brazilian public health system. The concepts of paradigm and normal science as well as Kuhn's conception of scientific learning are consistent with Fleck's concepts of thought style' and initiation in the esoteric contexts of a thought collective'. Both theoreticians reinforce themselves mutually with regard do the pertinence of applying these categories to the field of medicine and medical education. They also point to the need of an early involvement of students in the practice of primary care and related abilities and practices, taught by professionals in these fields. Moreover, these theories favor recognition and management of "paradigmatic or stylist reductions" besides increasing the clinical abilities and ethical performance of professionals and students. 


\section{INTRODUÇÃO}

O ensino médico brasileiro está no início de um processo de mudanças profundas e há muito necessárias. Após anos de críticas, comissões e debates, iniciativas de reformas curriculares com apoio federal ocorrem em escolas médicas. O ensino médico esteve, até hoje, centrado nos ambientes hospitalares e sob o domínio das especialidades médicas. Permaneceu eminentemente voltado para a formação de profissionais protoespecialistas, dirigidos para uma prova de residência em alguma especialidade médica.

Os valores, os exemplos, os conteúdos, as experiências determinantes ou mais marcantes por que passam os alunos neste contexto os transformam em médicos pouco aptos a trabalharem com criatividade, satisfação e competência na atenção primária do Sistema Único de Saúde (SUS).

Em paralelo, o SUS vem conseguindo priorizar na sua organização a atenção primária, ou rede básica, por meio de aperfeiçoamentos no sistema de gestão e da estratégia "Saúde da Família". A atenção primária, aos poucos, vai se constituindo como a base fundamental e o locus principal da atenção à saúde no SUS, ganhando espaço no mercado de trabalho e vivendo as agruras de uma herança difícil: há poucos profissionais engajados e competentes para o trabalho na atenção primária e na Saúde da Família, relativamente nova e em construção, como de resto é o próprio SUS.

Por outro lado, as experiências de transformação curriculares voltadas para a formação de profissionais para o SUS enfrentam agruras similares: não existem também docentes qualificados para formar esses profissionais.

Uma parte da epistemologia recente, chamada póspositivista, tem vários autores que fornecem, por hipótese, conceitos, idéias e direções para a formação médica, dentre os quais se destacam Thomas Kuhn e Ludwik Fleck. Esses autores descreveram, analisaram e valorizaram aspectos pedagógicos, psicossociais e cognitivos envolvidos na iniciação nas disciplinas e práticas científicas ou especializadas com notável convergência, sendo o segundo, de certo modo, precursor do primeiro.

O objetivo deste artigo é sintetizar alguns aspectos das idéias de Kuhn e Fleck, compará-los e correlacioná-los à atividade médica, para extrair deles contribuições para a mudança do ensino médico. Trata-se de uma síntese de pesquisa teórica ${ }^{1,2}$, escrutinada pela prática clínica e de ensino do autor.

Inicialmente, Kuhn e Fleck são introduzidos por intermédio de uma síntese livre de suas idéias; em seguida, estas são correlacionadas com a medicina e o ensino médico e alguns desafios atuais de sua reforma, obviamente sem pretender esgotar o tema.

\section{KUHN: PARADIGMAS, CIÊNCIA NORMAL E INICIAÇÃO}

A epistemologia de Thomas Kuhn, físico norte-americano que enveredou pelo estudo da história e filosofia da Ciência, tem uma dupla originalidade: tenta caracterizar a própria atividade de investigação e não seus produtos ou uma imagem idealizada do que deveria ser ${ }^{3}$. Dentre outros, Kuhn é considerado um divisor de águas na epistemologia, marco da chamada epistemologia histórico-interpretativa, ou pós-positivista ${ }^{4}$.

Segundo Kuhn ${ }^{5,6,7}$, grosso modo, os paradigmas seriam um conjunto de concepções, modelos, valores, padrões perceptivos e práticas consensualmente aceitos em uma comunidade científica, os quais guiam a prática do cientista ${ }^{8}$. $O$ paradigma não foi definido precisamente por Kuhn, motivo pelo qual foi muito criticado 9 . Pode-se considerá-lo uma visão do mundo expressa numa teoria ou subjacente a ela, ou como um mapa de referência, ou como um conjunto de regras implícitas, pressupostos e modos perceptivos com sua respectiva cognição que guiam a atividade do cientista.

$\mathrm{O}$ autor refinou posteriormente o conceito, reconhecendo seu uso em dois sentidos: um mais amplo, que chamou "matriz disciplinar", a qual engloba a maior parte ou todos os objetos de empenhamento da comunidade científica a ela adscrita, consistindo em quatro elementos principais: generalizações simbólicas*, pressupostos metafísicos, valores e exemplos partilhados de soluções de problemas concretos: os exemplares. Estes últimos são um componente do conhecimento relacionado a "uma percepção aprendida de semelhança", aplicada no modo de enquadramento e resolução de enigmas, a qual é tanto lógica quanto psicologicamente anterior a qualquer critério e se baseia em exemplos partilhados que podem desempenhar funções cognitivas comumente atribuídas a regras - e aos quais o autor dá grande importância, constituindo o sentido mais restrito e mais inovador do sentido do termo ${ }^{4,7}$.

Kuhn sustenta que a Ciência progride quando os cientistas são treinados numa tradição intelectual comum e usam essa tradição para resolver os problemas que ela suscita. Vê a história de uma ciência "madura" como sendo, essencialmente, uma sucessão de tradições, cada uma das quais com sua própria teoria e seus próprios métodos de pesquisa, cada uma guiando uma comunidade de cientistas durante certo período de tempo (ciência normal) e sendo finalmente abandonada após uma crise paradigmática que gera uma revolução científica, coisa muito rara. Crise essa que proporciona certo tempo de ciência revolucionária, que, por sua vez, engendrará um novo paradigma que se estabilizará e permitirá nova fase normal ${ }^{10}$.

Um dos maiores sinais da estabilização de um paradigma é o estabelecimento de conjuntos teórico-metódológicos que

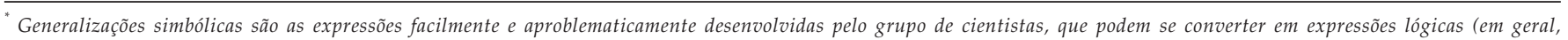

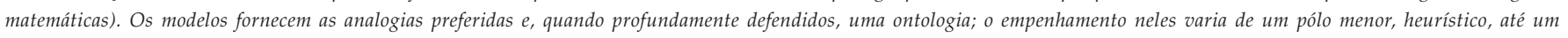
empenhamento maior, metafísico. 
deixam de ser debatidos e logram monopolizar, como padrãoouro, a atividade de pesquisa, sem rivais significativos.

Segundo Kuhn", o cientista normal resolve "quebra-cabeças" e enigmas que colocam em teste a habilidade dos indivíduos na solução de problemas. Se a resolução não for exitosa, são a perícia e as hipóteses do cientista que fracassam, não o conjunto das regras (o paradigma). Assim, os problemas não são definidos estritamente por sua relevância, mas por sua capacidade de serem solucionáveis.

Um paradigma seleciona partes da realidade relacionandoas e explicando o mundo. Mas deixa outras partes e fenômenos, ou suas relações, "de fora". Por vezes, tais fenômenos ou relações não previstos ou inexplicados aparecem aos cientistas, constituindo o que Kuhn chamou de "anomalias" (como, por exemplo, a subjetividade dos doentes no paradigma da biomedicina $\left.^{8}\right)$. E suscitam várias reações, sendo as mais comuns: $1^{\mathrm{o}}$ : deve haver algum erro, há que repetir tudo de novo; $2^{\circ}$ : o que é que vão dizer se lhes contar o dado que obtive? É melhor ficar quieto; 3: é melhor esconder as provas (muito comum). Caso

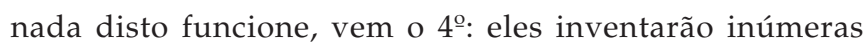
articulações e modificações ad hoc de sua teoria a fim de eliminar qualquer conflito aparente ${ }^{11}$ (p.192-3).

A estrutura da mudança do saber científico em Kuhn denota uma idéia de progresso, em que ele não é visto como uma progressão para o que seria a "Verdade" ou a "descrição objetiva da natureza". O essencial consiste em não haver progresso dirigido para um fim, mas tão-somente a partir de uma origem primitiva ${ }^{3}$.

Outra idéia kuhniana, a incomensurabilidade entre paradigmas, significa que cada paradigma seleciona e articula distintos aspectos da realidade, apreendendo e explicando a mesma de forma particular, construindo "um mundo". Esse "mundo" é diferente de outro, construído por outro paradigma. Isso pode ser compreendido por meio da analogia com as figuras que se transformam conforme a gestalt visual de quem as vê: ou se vê uma velha ou se vê uma jovem com um lindo colar, mas não se vê as duas ao mesmo tempo ${ }^{7}$. As linhas desenhadas são as mesmas, mas as figuras são diferentes: cada paradigma faz seu praticante viver num mundo diferente.

Um ponto importante é o modo como o aprendiz aprende a ser cientista. Segundo Kuhn' ${ }^{6}$, esse modo é baseado nos exemplos dos seus professores e nos manuais em que estuda, onde as contradições entre teorias são obscurecidas, e uma linha de progresso lógico é sutilmente estabelecida entre as antigas teorias e as contemporâneas. Isso mostra o caráter coletivo da construção do conhecimento e uma semelhança entre a ciência e outras tradições de saber e prática especializados. Os exemplares, largamente utilizados, são formas direcionadoras e constrangedoras da percepção e da validação de perguntas e das soluções que não seguem racionalidade alguma, são simplesmente impostos no processo de iniciação.

O aprendizado científico é um aprender de habilidades manuais, perceptivas e cognitivas de forma dirigida. A ciência normal, assim, é vista como mais dogmática do que parece, mais destinada a reafirmar as suposições básicas do paradigma do que a solucionar relevantes problemas do mundo ou a responder perguntas fundamentais sobre sua natureza ou, na área da saúde, sobre os adoecimentos e cura dos doentes.

Sendo eminentemente auto-referenciada, a comunidade científica e sua cultura ganham um peso antes negligenciado na construção e legitimação do conhecimento ${ }^{3}$. Lendo uma literatura restritíssima, que circula apenas internamente, desobrigados de explicar ou responder a quaisquer questionamentos ou opiniões vindos de fora de seu universo técnico (que por definição é um ambiente incompetente), os cientistas vivem num mundo peculiar. Constroem uma visão de mundo, um mundo próprio, legitimado e sancionado como verdade pela posição mitológica da Ciência como produtora de verdades na modernidade.

\section{CIÊNCIA, PARADIGMA E ATIVIDADE MÉDICA}

A atividade médica não é, evidentemente, apenas uma atividade científica, mas é intensamente influenciada por esta e cada vez mais. Ela é, em parte, científica, ou ao menos busca sêlo em vários momentos: a atividade diagnóstica, por exemplo, é tipicamente semelhante à científica e por ela progressivamente regrada. Cada doente se converte em um enigma: descobrir qual a doença que nele se apresenta, para então eliminá-la1,12.

Embora o conceito kuhniano tenha sido forjado numa perspectiva histórica, retrospectiva e restrita à produção do saber, e assim, a rigor, somente no futuro poderíamos caracterizar um paradigma para o saber da biomedicina dos séculos 20 e 21, tem sido fértil e produtiva uma extensão do conceito para o presente momento e para a prática médica, tornando possível uma descrição de algumas características centrais de tal paradigma.

Vários estudos, usos e analogias das relações entre medicina e Ciência usaram o conceito kuhniano, caracterizando o desenvolvimento de um paradigma biomecânico, ou mecanicista, ou cartesiano, vigente na biomedicina ${ }^{1,2,12-18}$. O uso se estendeu à saúde, inclusive na área da saúde pública ${ }^{19-22}$, epidemiologia ${ }^{23}$ e aprendizado de medicinas exóticas ${ }^{24}$.

A produção de saber na biomedicina, durante o século 20, estabilizou-se em termos teóricos e metodológicos. Um dos sinais evidentes disso é a estabilização sem questionamentos de padrões-ouro metodológicos, como os ensaios clínicos controlados, por exemplo, a imperar consensualmente. $\mathrm{O}$ paradigma biomecânico parece vigorar plenamente, agora centrado na biologia molecular e na genética como eixos centrais 
de detalhamento. Ele é norteado por uma visão ontológicolocalista e mecânico-causal das doenças ${ }^{25}$, sendo centrado eminentemente nestas "entidades", tanto na produção de conhecimento quanto na prática médica.

As doenças não se encontram conceituadas teoricamente em local algum, mas algumas representações hegemônicas sintetizam sua concepção neste paradigma: as doenças "são coisas, de existência fixa e imutável, de lugar para lugar e de pessoa para pessoa; as doenças se expressam por um conjunto de sinais e sintomas, que são manifestações de lesões, que devem ser buscadas por sua vez no âmago do organismo e corrigidas por algum tipo de intervenção concreta"12 (p. 117). Um biologicismo materialista, que procura causas micromateriais como causas únicas no corpo para explicar os adoecimentos, e intervir sobre eles pode ser considerado o carro-chefe deste paradigma.

O ensino da biomedicina envolve a iniciação numa tradição de curadores, a corporação médica, e num agregado disciplinar de disciplinas biológicas, clínicas e epidemiológicas ${ }^{12}$, num local típico que tem concretizado esta iniciação: o hospital. Local onde os exemplares, os saberes, os valores e as práticas serão absorvidos e reproduzidos.

São evidentes os problemas pedagógicos dessa ambiência: ali, onde há iminente risco de vida, onde o biológico é o que conta, onde o poder sobre o paciente é quase total, onde o diálogo quase inexiste, onde a vida real do doente no "seu mundo existencial e psicossocial" está entre parênteses e pouco importa, onde o esquartejamento dos doentes está institucionalizado pelas especialidades, é que o aluno aprenderá a ser um médico. Muitas vezes arrogante, indelicado, seco, autoritário, biologicista, controlador, intervencionista.

Como síntese geral, a idéia de paradigma (estendida ao presente e à prática médica) permite compreender a coerência paradigmática dos médicos, maravilhosa em algumas situações, triste em muitas outras.

\section{A EPISTEMOLOGIA DE LUDWIK FLECK}

A obra principal de Fleck ${ }^{26}$, médico e cientista da década de 1930, herdeiro de uma tradição ou "escola" de médicos filósofos da Polônia ${ }^{27}$, traz uma análise da construção dos fatos e saberes científicos, associados ao que o autor chamou de estilos de pensamento, que estão vinculados aos coletivos de pensamento.

Um estilo de pensamento "é uma coerção determinada de pensamento e a totalidade da preparação e disponibilidade intelectual orientada a ver e atuar de uma forma e não de outra" (p. 111) ${ }^{26}$. "Coage os indivíduos e determina "o que não pode pensar-se de outra forma"26 (p. 145).

Ou ainda: estilo de pensamento é um modo de ver, entender e conceber, processual, dinâmico e sujeito a mecanismos de regulação, determinado psico-socio-histórico-culturalmente, que leva a um corpo de conhecimentos e práticas compartilhado por um coletivo com formação específica ${ }^{28}$.

O estilo de pensamento unifica as concepções, valores, crenças e práticas de um coletivo de pensamento, que se organiza em círculos hierarquizados epistemologicamente: os círculos centrais esotéricos, constituídos pelos produtores do saber especializado; círculos intermediários, reprodutores e praticantes deste saber; e, por fim, os círculos mais periféricos, exotéricos: os leigos, que utilizam técnicas e saberes dos anteriores.

Segundo Fleck ${ }^{26}$, toda a base de um saber especializado deriva da cultura geral, dos estratos exotéricos, que fornecem o senso de confiança, as concepções gerais de mundo e de universo, a estabilidade emocional das certezas evidentes por si sós. Algumas partes desse saber são reconstruídas pelos círculos esotéricos de um estilo de pensamento, escrutinadas pelos interesses, métodos, valores e técnicas desenvolvidos por esses círculos, gerando novos saberes, tecnologias e práticas sociais, que fazem o caminho de volta ao mundo exotérico e o influenciam. Nesse caminho de volta, os saberes de novo se transformam, ficando mais simples, mais dogmáticos e mais esquemáticos.

Nas medicinas, os doentes são os círculos exotéricos, os clínicos ou curadores são os intermediários, e os "superespecialistas" (na biomedicina, os cientistas) são os esotéricos.

No desenvolvimento dos fatos, há um intercâmbio e uma constante troca de posição entre o que Fleck ${ }^{26}$ chamou de conexões ativas e conexões passivas do conhecimento. As pressuposições respondem pela conexões ativas e formam a parte do saber que pertence ao coletivo. Os resultados obtidos equivalem às conexões passivas e formam o que se percebe como realidade objetiva. Para Fleck ${ }^{26}$, os elementos passivos e ativos não são separáveis completamente, nem logicamente, nem historicamente.

A construção do conhecimento científico dá-se por um processo coletivo de transformação das conexões ativas em passivas e vice-versa, a partir de proto-idéias presentes na cultura geral. Os estilos de pensamento, à semelhança dos paradigmas de Kuhn, tendem a centrar-se nos pressupostos, teorias e métodos cujas conexões ativas e passivas são selecionadas de modo a manter a harmonia e a coerência no sistema de idéias e destas com as conexões passivas. Em outras palavras, as conexões ativas que não são harmônicas com o estilo de pensamento, ou as conexões passivas que não se harmonizam com as ativas do estilo tendem a ser descartadas e negadas ou são reinterpretadas².

Fleck considera que, ao longo do processo de desenvolvimento do conhecimento, podem ocorrer duas fases: 
uma "clássica" ou de "extensão do estilo", em que a harmonia de suas ilusões impera inconteste; e outra de "complicações", em que conexões passivas estranhas e exceções (ou anomalias, cf. Kuhn) começam a aparecer por demais e a questionar o saber estabelecido, podendo mudá-lo - em paralelo com as fases de ciência normal e revolucionária de Kuhn²9.

Outro paralelo com Kuhn é que entre estilos de pensamento estranhos muitas vezes pouca comunicação é possível, e, segundo Fleck, uma incomensurabilidade ou incongruência vigora ${ }^{29}$.

Por último, também de forma semelhante a Kuhn, Fleck ${ }^{26}$ analisa a iniciação num estilo de pensamento científico ou médico como progressão na direção esotérica de forma semelhante a qualquer iniciação: há rituais e pedagogias que constituem uma "suave coerção" e imposição dos saberes e práticas aceitos, permeadas por forças psicossociais envolvidas no processo de aceitação e adaptação do neófito nos círculos mais esotéricos, que não tem a ver com critérios racionais.

\section{FLECK, KUHN E A REFORMA DO ENSINO MÉDICO}

O conceito de estilo de pensamento proposto por Fleck refere-se à Ciência. No entanto, não é restrito a ela, pelo contrário: os estilos e os coletivos de pensamento transcendem o locus social científico, podem ser identificados em outras instâncias sociais e culturais. Neste sentido, os estilos de Fleck são conceituados de forma mais ampla e genérica do que os paradigmas de Kuhn. Essa generalidade dos estilos de pensamento e sua semelhança com os paradigmas indicam a possibilidade, propriedade e pertinência da expansão do conceito kuhniano para além de sua formulação estrita.

Se o uso do modelo kuhniano foi estendido e extrapolou os limites da história das ciências naturais, o uso das categorias fleckianas é mais recente e exígua. No Brasil, autores como Koifman ${ }^{30}$, Delizoicov ${ }^{31}$, Backes ${ }^{32}$, Da $\operatorname{Ros}^{27}$, Cutolo ${ }^{28}$, Camargo $\mathrm{Jr}^{12}$ e Tesser ${ }^{2,33}$ utilizaram os conceitos fleckianos em pesquisas sobre ensino e prática de medicina, enfermagem e biologia, bem como mudanças curriculares e saúde pública. Em outros lugares, autores aplicaram suas categorias a estudos tão diferentes como a história do conceito de sífilis ${ }^{26}$, a historiografia da ciência ${ }^{34} \mathrm{e}$ relações entre coletividades em pesquisas ${ }^{35}$.

Algumas considerações gerais podem ser feitas sobre o ensino médico, tomando-se por pressuposto que existe na biomedicina um estilo de pensamento amplo largamente dominante (que contém diferenciações e matizes dentro dele), correspondente ao que chamei de paradigma biomecânico.

Primeiro, há que compreender que, se os alunos aprendem o que seus professores fazem por exemplos, estes só podem ensinar o que efetivamente praticam. Se praticam medicina hospitalar e especializada, é isso que os alunos aprendem dentro do hospital. E continuarão aprendendo se ficarem estudando lá. Sua iniciação na cultura e no clima relacional do hospital, frio, técnico, centrado nas doenças e autoritário, faz com que estas características sejam introjetadas inconscientemente nos alunos, no processo de aceitação pelos pares e de identificação com os mestres, e nas práticas cotidianas, como transformar doentes em números de leitos ou em síndromes e sinais cujo aprendizado perceptivo está se dando ("Há uma esplenomegalia no leito X", "um sopro diastólico na enfermaria Y", etc.).

Se a prioridade é a formação médica para a construção de um SUS e de uma medicina cada vez melhores, ela aponta para a formação do médico geral ou generalista, e a ambiência adequada para essa formação é o local onde ocorre de fato tal prática, ou seja, a própria rede básica e as equipes de PSF.

Nessa lógica, os exemplares das propostas de mudança devem sair dos hospitais, deslocando-se para uma ambiência onde ocorram melhores práticas-modelo que iniciem os alunos na vida médica ambulatorial do SUS. Isto é, para a própria rede ambulatorial do SUS, principalmente a atenção primária e, recentemente, o PSF, para que os aprendizes possam aprender como bem trabalhar ali. Tanto melhor se um outro paradigma estivesse ali já constituído, mas arrisco afirmar que, por enquanto, isso ainda não ocorre.

Assim, está-se autorizado a pensar que a reforma do ensino médico deve envolver uma inversão na ambiência, em médio prazo: o hospital se tornará um estágio por onde se passa, e a prática ambulatorial generalista será o locus principal onde se aprende medicina, da teoria e da anamnese ao tratamento e acompanhamento dos doentes.

Logo, há que se esboçar um protótipo ideal de profissional docente e de ambiente profissional para os estudantes terem como exemplo e modelo. Se é um médico geral ou generalista crítico, humanista, competente, ético e habilitado para o trabalho na rede básica do SUS que se quer formar, deve haver docentes com esses atributos trabalhando em serviços do SUS para ensinar os aprendizes. Mas para ser otimista, atualmente, há raríssimos destes médicos em posição de docência que praticam, e assim podem ensinar, boa medicina em serviços de atenção primária ou equipes de PSF.

Essas ponderações reforçam a direção recente da política do SUS de induzir a formação de pessoal para si próprio e de pressionar as escolas médicas no sentido de ambientar o mais precocemente possível os alunos em serviços de atenção primária e ambulatorial para aprendizagem prática do que ali ocorre.

No processo de operacionalização dessas mudanças, também é previsível, e já vem acontecendo, que uma longa fase transitória se desenrole, sem docentes competentes e com conhecimento da 
prática médica generalista na atenção primária. Por esse motivo, um duplo movimento cheio de ruídos e atritos tem sido necessário: transformar os "melhores" dentre os médicos do SUS e do PSF próximos aos centros formadores em tutores ou supervisores de estágios de alunos de Medicina; e, ao mesmo tempo, introduzir, aos poucos, docentes relativamente dispostos ao aprendizado na rede básica, para que aprendam com a realidade e junto com seus colegas generalistas a difícil arte e técnica de ser um médico geral ou de família, para que possam ensinar isso a seus alunos e construir uma tradição e um estilo de pensamento de excelência, calcados sobre a atenção ambulatorial no PSF e na rede básica.

Outra ordem de contribuições de Kuhn e Fleck é mais epistemológica e ética: o reconhecimento dos estilos de pensamento ou paradigmas contribui para a tomada de consciência e o manejo das chamadas "reduções paradigmáticas" ${ }^{\prime 36}$.

A redução paradigmática refere-se ao fato de que a abordagem de qualquer fenômeno orientada por um paradigma gera um saber que diz respeito ao fenômeno sempre de forma incompleta, parcial e interessada. Mas ao explicá-lo e lhe dar sentido, o paradigma acaba gerando outro fenômeno similar e, subliminarmente, um outro mundo, que não é mais o mundo cru do fenômeno inicial, mas, sim, uma seleção de alguns aspectos desse fenômeno e seu mundo ligados aos pressupostos, métodos, aspirações e valores do paradigma ou estilo. Nesse processo, cria um objeto artificial, científico, que tende a roubar a cena. No caso da biomedicina, as entidades doenças ou patologias.

Percebendo a duplicidade do mundo dos fenômenos - por um lado, um mundo de fenômenos relativamente crus, complexo, repleto e misterioso, trazido pelos pacientes; por outro, um mundo reduzido por um paradigma, simplificado, com menos elementos, com relações supostamente mais claras entre eles -, um mundo de patologias, síndromes, órgãos e sistemas, pode-se manter mais facilmente a duplicidade dos dois mundos, o cru (o adoecimento do doente) e o reduzido (a doença do médico): considerar o primeiro como o desafio prioritário e missão ética essencial do curador, e o segundo como instrumento para a difícil execução dessa primeira missão.

Com isso, a consciência da missão ética de curador, referida às pessoas doentes, fica reforçada, minimizando o perigo da ontologização inconsciente das patologias (que passam, assim, a ser objeto primordial da atenção médica, degradando tanto a relação curador-doente quanto a autoimagem do médico, reduzindo esta última à de um técnico "cientista normal" -, diagnosticador e prescritor, quando precisa ser muito mais que isso).
Tratou-se aqui de sintetizar algumas idéias epistemológicas de Kuhn e Fleck, que parecem ser valiosos instrumentos de compreensão dos processos de construção de saber e práticas e de iniciação dos médicos. Outras explorações podem ampliar e detalhar sua contribuição, e os beneficiários de sua assunção pelas reformas da formação médica serão certamente os usuários dos serviços de saúde e os doentes, razão da existência das medicinas e escolas médicas.

\section{REFERÊNCIAS}

1. Tesser CD. A biomedicina e a crise da atenção à saúde: um ensaio sobre a desmedicalização. Campinas, SP; 1999. Mestrado [Dissertação] - Universidade Estadual de Campinas.

2. Tesser CD. Epistemologia contemporânea e saúde: a luta pela verdade e as práticas terapêuticas. Campinas, SP; 2004. Doutorado [tese] - Universidade Estadual de Campinas.

3. Stengers I. A descrição da atividade científica por T.S. Kuhn. In: Carrilho MM, org. História e prática das ciências. Lisboa: A Regra do Jogo; 1979. p.78-116.

4. Jacobina RR. O paradigma da epistemologia histórica: a contribuição de Thomas Kuhn. Hist Cienc Saude. 19992000; 6(3): 609-30.

5. Kuhn TS. Reflexões sobre meus críticos. In: Lakatos I, Musgrave A, orgs. A crítica e o desenvolvimento do conhecimento. São Paulo: Cultrix; 1979. p.285-343.

6. Kuhn TS. A estrutura das revoluções científicas. 2 ed. São Paulo: Perspectiva; 1987.

7. Kuhn TS. A tensão essencial. Lisboa: Ed. 70; 1989.

8. Guedes CR, Nogueira MI, Camargo Júnior KR. A subjetividade como anomalia: contribuições epistemológicas para a crítica do modelo biomédico. Cienc Saude Col. [online]. 2006. (No prelo). [capturado 22 set. 2006]; Disponível em: http://www.abrasco.org.br/ c i e n c i a e s a d e coletiva / a r t i gos/ artigo_int.php?id_artigo=18.

9. Masterman M. A natureza do paradigma. In: Lakatos I, Musgrave A. A crítica e o desenvolvimento do conhecimento. São Paulo: Cultrix; 1979.

10. Kneller GF. A ciência como atividade humana. Rio de Janeiro: Zahar; 1980.

11. Alves R. Filosofia da ciência: introdução ao jogo e suas regras. 21 ed. São Paulo: Brasiliense; 1995.

12. Camargo Júnior KR. Biomedicina, ciência \& saber: uma abordagem crítica. São Paulo: Hucitec; 2003.

13. Dossey L. Espaço, tempo e medicina. São Paulo: Cultrix; 1998. 
14. Capra F. O ponto de mutação. São Paulo: Cultrix; 1980.

15. Queiroz MS. O paradigma mecanicista da medicina ocidental moderna: uma perspectiva antropológica. Rev Saude Publica. 1986; 20(4): 309-17.

16. Camargo Júnior KR. Paradigmas, ciência e saber médico. Rio de Janeiro: IMS/UERJ; 1992. (Série estudos em saúde coletiva, 6).

17. Mattos RA, Vianna CMM, Camargo Júnior KR. Paradigmas, ciência e saber médico: uma discussão. Rio de Janeiro: IMS/UERJ; 1993. (Série estudos em saúde coletiva, 31).

18. Schramm FR. Paradigma biotecnocientífico e paradigma bioético. In: ODA LM, org. Biosafety of transgenic organisms in human health products. Rio de Janeiro: Fiocruz; 1996. p.109-127.

19. Mendes EV. A evolução histórica da prática médica: suas implicações no ensino, na pesquisa e na tecnologia médicas. Belo Horizonte, MG: PUC-MG/Finep; 1984.

20. Kisil M. Educação em administração de saúde na América Latina: a busca de um paradigma. São Paulo: USP; 1994.

21. Scherer MDA, Marino SRA, Ramos FRS. Rupturas e resoluções no modelo de atenção à saúde: reflexões sobre a estratégia saúde da família com base nas categorias kuhnianas. Interface. 2004-2005; 9(16): 53-66.

22. Luz MT. Cultura contemporânea e medicinas alternativas: novos paradigmas em saúde no fim do século XX. Physis. 1997; 7(1): 13-43.

23. Almeida-Filho N. Os paradigmas da epidemiologia. In: Almeida-Filho N, org. A clínica e a epidemiologia. Rio de Janeiro: Abrasco; 1992. p.90-104.

24. Nogueira MI. Entre a conversão e o ecletismo: de como médicos brasileiros tornam-se "chineses". Rio de Janeiro; 2003. Doutorado [Tese] - Universidade do Estado do Rio Janeiro.

25. Almeida EV. As razões da terapêutica. Rio de Janeiro; 1996. Doutorado [Tese] - Universidade do Estado do Rio de Janeiro.

26. Fleck L. La génesis y el desarrollo de um hecho científico: introducción a la teoría del estilo de pensamiento y del colectivo de pensamiento. Madrid: Alianza Editorial; 1986.

27. Da Ros MA. Estilos de pensamento em saúde pública: um estudo da produção da FSP-USP e ENSP-FIOCRUZ, entre
1948 e 1994, a partir da epistemologia de Ludwik Fleck. Florianópolis,SC; 2000. Doutorado [Tese] - Universidade Federal de Santa Catarina.

28. Cutolo LRA. Estilo de pensamento em educação médica: um estudo do currículo do curso de graduação em medicina da UFSC. Florianópolis, SC; 2001. Doutorado [Tese] - Universidade Federal de Santa Catarina.

29. Delizoicov D, Castilho N, Cutolo LRA, Da Ros MA, Lima AMC. Sociogênese do conhecimento e pesquisa em ensino: contribuições a partir do referencial Fleckiano. In: II Encontro Nacional de Pesquisas em Ensino de Ciências ENPEC; 1999; Valinhos, SP.

30. Koifman L. A crítica ao modelo biomédico na reformulação curricular do curso de medicina da Universidade Federal Fluminense. Rio de Janeiro; 1996. Mestrado [Dissertação] - Fundação Oswaldo Cruz.

31. Delizoicov NC. Os professores de ciências naturais e o livro didático. Florianópolis, SC; 1995. Mestrado [Dissertação] - Universidade Federal de Santa Catarina.

32. Backes VMS. O legado histórico do modelo Nightngale: seu estilo de pensamento e sua práxis. Florianópolis, SC; 1997. Doutorado [Tese] - Universidade Federal de Santa Catarina. (mimeo).

33. Tesser CD. Medicalização social (I): o excessivo sucesso do epistemicídio moderno na saúde. Interface. 2006; 10(19): 61-76.

34. Lowy I. Ludwik Fleck e a presente história das ciências. Hist Cienc Saude. 1994; 1(1): 7-18.

35. Oudshoorn N. On the making of sex hormones: research materials and the production of knowledge. Soc Stud Sci. 1990; 20(1): 5-33.

36. Fourez G. A construção das ciências: introdução à filosofia e à ética das ciências. São Paulo: Ed. UNESP; 1995.

\section{CONFLITOS DE INTERESSE:}

Declarou não haver.

\section{ENDEREÇO PARA CORRESPONDÊNCIA:}

Charles Dalcanale Tesser

Rua Laureano 970, Campeche

88065-040 - Florianópolis - SC

E-mail: charlestesser@ccs.ufsc.br 